\title{
Charge accumulation in DC cables: a macroscopic approach
}

\author{
McAllister, lain Wilson; Crichton, George C; Pedersen, Aage
}

Published in:

Conference Record of the IEEE International Symposium on Electrical Insulation

Link to article, DOI:

10.1109/ELINSL.1994.401528

Publication date:

1994

Document Version

Publisher's PDF, also known as Version of record

Link back to DTU Orbit

Citation (APA):

McAllister, I. W., Crichton, G. C., \& Pedersen, A. (1994). Charge accumulation in DC cables: a macroscopic approach. In Conference Record of the IEEE International Symposium on Electrical Insulation (pp. 212-216). IEEE. https://doi.org/10.1109/ELINSL.1994.401528

\section{General rights}

Copyright and moral rights for the publications made accessible in the public portal are retained by the authors and/or other copyright owners and it is a condition of accessing publications that users recognise and abide by the legal requirements associated with these rights.

- Users may download and print one copy of any publication from the public portal for the purpose of private study or research.

- You may not further distribute the material or use it for any profit-making activity or commercial gain

- You may freely distribute the URL identifying the publication in the public portal 
Conference Record of the 1994 IEEE International Symposium on Electrical Insulation, Pittsburgh, PA USA, June 5-8, 1994

Charge Accumulation in DC Cables: A Macroscopic Approach

\author{
I.W. McAllister and G.C. Crichton \\ A. Pedersen \\ Electric Power Engineering Department \\ Building 325 \\ Physics Department \\ DK-2800 Lyngby, Denmark.
}

\begin{abstract}
The accumulation of space charge in solid dielectrics is examined from the macroscopic point of view using electromagnetic field theory. For practical dielectrics, it is shown that the occurrence of such charges is an inherent consequence of a non-uniform conductivity. The influence of both temperature and electric field strength upon this space charge formation is elucidated. Thereafter the theory is applied to DC cables.
\end{abstract}

\section{INTRODUCTION}

The field distribution in a DC cable is controlled by the conductivity of the insulating dielectric. This conductivity is a rapidly increasing function of temperature and to a lesser degree also of field strength. Such a non-linear characteristic implies the existence of space charges within the bulk dielectric [1]. If the functional dependence of the conductivity with temperature and field strength is known the field distribution can be evaluated correctly within the framework of circuit analysis. This approach is implemented in many papers and textbooks on cable technology. Although space charges are in fact inherently included in such an approach, their existence is eclipsed by the rituals of circuit theory. Moreover, it is evident that many of these authors are unaware of the underlying phenomenon of space charge accumulation.

Space charge accumulation in solid dielectrics has been addressed directly in numerous recent publications in which the analysis is based on a molecular interpretation of conduction in dielectrics. A side effect of this emphasis on the molecular aspects appears to be a disregard of the macroscopic side of the problem. It apparently arouses little interest that any macroscopic piece of dielectric must obey the laws of classical electromagnetism as expressed in Maxwell's equations and that, is a consequence, a field theoretical approach must provide essential insight into the problem of charge accumulation.

In the present study space charge accumulation in dielectrics is addressed in terms of classical electromagnetic field theory. General expressions are deduced for the build-up of volume and interface space charges. Subsequently, specific expressions for the accumulation of volume space charge in DC cables are derived. The particular influence of both temperature and field strength upon the accumulation of such space charge is brought into focus.

CHARGE ACCUMULATION IN LOSSY DIELECTRICS

In a Laplacian field the divergence of the electric flux density $\vec{D}$ is everywhere zero. Any deviation from the Laplacian distribution requires that somewhere the divergence of $\vec{D}$ must differ from zero, and that consequently a space charge distribution must exist in the dielectric. The volume density $\rho$ of this distribution is governed by Maxwe11's third equation; viz.

$\rho=\operatorname{div} \vec{D}$

Since even the best dielectric possesses some degree of conductivity, an electric 
field of strength $\vec{E}$ in the bulk material will establish a volume current density $\vec{J}$ given by

$$
\vec{J}=\boldsymbol{\gamma} \vec{E}
$$

where $\gamma$ is the conductivity. We can therefore rewrite (1) in the form

$$
\rho=\operatorname{div}\left(\frac{\varepsilon}{\gamma} \vec{J}\right)
$$

or

$\rho=\frac{\varepsilon}{\gamma} \operatorname{div} \vec{J}+\vec{J} \cdot \operatorname{grad}\left(\frac{\varepsilon}{\gamma}\right)$

in which $\varepsilon$ denotes the absolute permittivity. The divergence of $\vec{J}$ is connected with the rate of change with time $t$ of the space charge density through the continuity equation: $1 . e$.

$\operatorname{div} \vec{J}+\frac{\partial \rho}{\partial t}=0$

Equation (4) therefore attains the final form

$\rho+\frac{\varepsilon}{\gamma} \frac{\partial \rho}{\partial t}=\vec{J} \cdot \operatorname{grad}\left(\frac{\varepsilon}{\gamma}\right)$

This is the basic differential equation which determines the growth of the space charge in both space and time.

\section{Steady-State Analysis}

In a DC field the current flow approaches a stationary condition, i.e. $\operatorname{div} \vec{J}=0$, for which the current density and the space charge density attain their final values. These are in view of (6) related by

$\rho=\vec{J} \cdot \operatorname{grad}\left(\frac{\varepsilon}{\gamma}\right)$

It is seen from (7) that a static space charge distribution in the bulk of the dielectric requires that a stationary current is flowing and that $\varepsilon / \gamma$ does not maintain a constant value throughout the dielectric. The former condition means that the dielectric system must be in direct contact with an external voltage source through electrodes. If this condition is not fulfilled the stationary electric field within a piece of solid dielectric placed in an external constant DC-field will approach zero, with the bulk of the dielectric becoming spacecharge-free. Depending on the relaxation time $r(=\epsilon(\gamma)$, this process may take a very long time and will be associated with a build-up of a surface layer of charges. The stationary surface charge density $\sigma$ is thus given by the $f l u x$ density $\vec{D}$ just outside the surface of the solid dielectric. Note that the stationary external electric field will be normal to this surface since the field inside the dielectric is zero. The static surface charge distribution is therefore the same as that on a conductor of the same geometrical form.

Dielectric Interfaces: Many dielectric systems contain interfaces between dielectrics with different permittivities and conductivities. Of special practical interest is a system in which the stationary current density is normal to the interface and no surface current is flowing in the system. This means that the stationary current density has the same value at the two sides of the interface. We refer to the two sides of the interface with the subscripts 1 and 2 . Let the stationary current density $J$ be directed from 1 to 2 . If $\varepsilon_{1} / \gamma_{1}$ differs from $\varepsilon_{2} / \gamma_{2}$ the electric flux densities at the two sides of the interface will have different values and a layer of space charge will consequently exist at the interface. This stationary surface charge density $\sigma$ is given by

$\sigma=D_{2}-D_{1}=\left[\frac{\varepsilon_{2}}{\gamma_{2}}-\frac{\varepsilon_{1}}{\gamma_{1}}\right] J$

A fuller discussion of such interface charge accumulation, with particular reference to GIS spacers, may be found in McAllister and Crichton [2].

Non-Linear Dielectrics: The conductivity, and to a lesser extent the permittivity, may be functions of several physical parameters. Let the number of these parameters be $n$ and let $p_{i}$ denote a parameter where $i$ can attain values from 1 to $n$. The gradient of $\varepsilon / \gamma$ is then given 
by

$\operatorname{grad}\left(\frac{\varepsilon}{\gamma}\right)=\sum_{i=1}^{n} \frac{\varepsilon}{\gamma}\left[\frac{1}{\varepsilon} \frac{\partial \varepsilon}{\partial p_{i}}-\frac{1}{\gamma} \frac{\partial \gamma}{\partial p_{i}}\right] \operatorname{grad} p_{i}$

By far the most important of these parameters $p_{i}$ are the temperature $T$ and the magnitude $|E|$ of the electric field. We will therefore restrict our analysis to the effects of $T$ and $|E|$. We can then consider the space charge density $\rho$ to be the sum of two components

$\rho=\rho_{T}+\rho_{|E|}$

where $\rho_{T}$ and $\rho_{|E|}$ are given by the expressions

$\rho_{T}=\frac{\varepsilon}{\gamma}\left[\frac{1}{\varepsilon} \frac{\partial \varepsilon}{\partial T}-\frac{1}{\gamma} \frac{\partial \gamma}{\partial T}\right] \vec{J} \cdot \operatorname{grad} T$

and

$\rho_{|E|}=\frac{\varepsilon}{\gamma}\left[\frac{1}{\varepsilon} \frac{\partial \varepsilon}{\partial|E|}-\frac{1}{\gamma} \frac{\partial \gamma}{\partial|E|}\right] \vec{J} \cdot \operatorname{grad}|E|$

The variation of $\varepsilon$ with either $T$ or $|E|$ is normally insignificant such that the influence of these differential parameters on $\rho_{T}$ and $\rho_{|E|}$ can be discarded in any discussion of cable insulation. In contrast, the conductivity increases rapidly with electric field strength and to an even greater degree with temperature. An exact physical theory for this dependence is however not available. Nevertheless it is possible to express experimental data in the form of empirical formulae which are valid over a limited range. A relationship especially suitable for a discussion of coaxial cables is the following [3];

$\gamma=\gamma_{a}\left[|E| /\left|E_{a}\right|\right]^{\nu} \exp \left[\alpha\left(T-T_{a}\right)\right]$

$\gamma_{a}$ is the conductivity for a reference field strength $E_{a}$ and reference temperature $T_{a}$. $\nu$ is a material constant which for oil-impregnated paper is approximately zero, but which for polyethylene can be ascribed values between 2.1 and 2.4 [3]. $\alpha$ is a constant and for the dielectrics used in cables may be given the value $0.1 \mathrm{~K}^{-1}$. From (13) we obtain

$\frac{\partial \boldsymbol{\gamma}}{\partial T}=\alpha \boldsymbol{\gamma}$

and

$\frac{\partial \gamma}{\partial|E|}=\left[\frac{\nu}{\left|E_{a}\right|}\left[\frac{|E|}{\left|E_{a}\right|}\right]^{-1}\right] \gamma$

\section{APPLICATION TO DC CABLES}

In the following we consider a coaxial cable insulated with a macroscopically homogeneous dielectric of constant permittivity. The cable has been on full load for a period of time such that the temperature and the electric field distributions represent stationary situations.

Under normal operating conditions, the heat generated in the insulation is negligible compared with the heat generated in the inner conductor. The temperature distribution is therefore given by

$\operatorname{div}[\kappa \operatorname{grad} T]=0$

If the thermal conductivity $\kappa$ of the dielectric can be considered to be constant then the temperature distribution is given by

$\frac{T_{a}-T}{T_{a}-T_{b}}=\frac{\ln (r / a)}{\ln (b / a)}$

in which $a$ and $b$ are the inner and outer radil of the dielectric, respectively. $T_{a}, T_{b}$ and $T$ are the temperatures at distances $a, b$ and $r$ from the axis. The temperature drop $\left(T_{a}-T_{b}\right)$ through the dielectric is related to the power dissipated in the conductor $P_{C}$ per length of cable in the following way

$T_{a}-T_{b}=\frac{\ln (b / a)}{2 \pi \kappa} P_{C}$

Insertion of (17) in (13) results in

$\gamma=\gamma_{a}\left[|E| /\left|E_{a}\right|\right]^{\nu}[r / a]^{-\beta}$ 
in which $\beta$ is a dimensionless quantity given by

$\beta=\alpha\left(T_{a}-T_{b}\right) / \ln (a / b)$

or

$\beta=\alpha P_{\mathrm{c}} / 2 \pi \kappa$

All vector quantities will because of the symmetry be directed either radially away from or towards the axis. We can therefore replace all vector equations with scalar equations. The direction of the field is away from the axis for $E$ positive, and is inherently identical in direction for the current density $J$.

As the current field is stationary, this implies that the product $r J(r)$ is a constant. Consequently by applying $\mathrm{Ohm}$ 's law (2), the stationary electric fleld can be determined: i.e. we have

$E=E_{a}\left[\gamma / \gamma_{a}\right]^{-1}[r / a]^{-1}$

where $E_{B}$ is the stationary field strength at the inner conductor. Insertion of (19) in (22) leads to the following expression for the stationary field distribution; viz.

$E=E_{a}[r / a]^{k-1}$

where $k$ is a constant given by

$k=\frac{\nu+\beta}{\nu+1}$

On introducing the applied DC voltage $U$, which is related to $E$ by

$U=\int_{a}^{b} E(r) \mathrm{d} r$

we find that

$E_{a}=\frac{k U}{a\left[(b / a)^{k}-1\right]}$

We now recall that the net space charge density $\rho$ is given by $\rho=\operatorname{div} \vec{D}$, which for a constant $\varepsilon$ can be expressed as

$\rho=\varepsilon\left[\frac{\partial E}{\partial r}+\frac{1}{r} E\right]$
This leads to

$\rho=\frac{\varepsilon k E_{a}}{a}[r / a]^{k-2}$

or in terms of the applied voltage

$\rho=\frac{\varepsilon k^{2} U}{a^{2}} \frac{[r / a]^{k-2}}{[b / a]^{k}-1}$

\section{DISCUSSION}

To investigate the manner in which the net space charge $\rho$ depends on the variations in temperature and electric field we apply (11) and (12) which, for a coaxial system and $\varepsilon$ considered as constant, attain the forms

$\rho_{T}=-\frac{\varepsilon}{\gamma^{2}} J \frac{\partial \gamma}{\partial T} \frac{\partial T}{\partial r}$

and

$\rho_{|E|}=-\frac{\varepsilon}{\gamma^{2}} J \frac{\partial \gamma}{\partial|E|} \frac{\partial|E|}{\partial r}$

From (17) we obtain

$\frac{\partial T}{\partial r}=-\frac{T_{a}-T_{b}}{\operatorname{rln}(b / a)}$

which combined with (14), (15), (19) and (24) leads to the following expressions for $\rho_{T}$ and $\rho_{|E|}$

$\rho_{T}=\frac{\varepsilon \beta E_{a}}{a}[r / a]^{k-2}$

$\rho_{|E|}=\frac{\varepsilon(k-\beta) E_{a}}{a}[r / a]^{k-2}$

It must be emphasized that although it is mathematically possible to resolve $\rho$ into two components, $\rho_{T}$ and $\rho_{|E|}$, these components do not individually represent space charge densities. It is the sum $\left(\rho_{T}\right.$ $\left.+\rho_{(E)}\right)$ which represents the existing space charge density. Nevertheless this division of $\rho$ into $\rho_{T}$ and $\rho_{|E|}$ is an essential step if the individual effects of the material parameters on the accumulation of space charge are to be 
elucidated. This aspect will form the subject of a future publication.

\section{CONCLUSION}

From a field-theoretical analysis
it is evident that space charge
accumulation in DC cables is an inherent
consequence of a non-uniform variation in
conductivity throughout the cable
insulation. This general deduction also
dispels the widespread belief that the
accumulation of space charge in DC cables
is uniquely linked to polymer insulation.

\section{REFERENCES}

[1] G.P. Harnwell, Principles of Electricity and Electromagnetism, (2nd ed.), New York: McGraw-Hill, 1949 .

[2] I.W. McAllister and G.C. Crichton, "Analysis of the Temporal Electric Fields in Lossy Dielectric Media", IEEE Trans. Elect. Insul. vol.26, pp.513-528, 1991 .

[3] V. Privezentsev, I. Grodnev, S. Kholodny and I. Ryazanov, Fundamentals of Cable Engineering, Moscow: Mir Publishers, 1973. 